\title{
Levator ani function before and after childbirth
}

\author{
*Ursula M. Peschers Assistant Lecturer, †Gabriel N. Schaer Senior Lecturer, \\ $\ddagger$ John O. L. DeLancey Associate Professor, *Bernhard Schuessler Professor \\ Departments of Obstetrics and Gynaecology at *Kantonsspital Luzern, Switzerland, †University Hospital, Zurich, Switzerland, and \\ $\ddagger$ The University of Michigan Medical Center, Ann Arbor, USA
}

Objective To evaluate pelvic floor muscle strength before and after vaginal birth.

Design Prospective repeated measures study.

Setting Main district hospital.

Population Fifty-five women: 25 primiparae and 20 multiparae following vaginal birth, and 10 women following elective caesarean delivery as a control group.

Methods Pelvic muscle strength was evaluated by palpation, perineometry and perineal ultrasound before childbirth in the 36 th to 42 nd week of pregnancy, three to eight days postpartum and six to ten weeks postpartum.

Main outcome measures Pelvic floor muscle strength on palpation, intravaginal squeeze pressure and vesical neck elevation during squeeze.

Results Pelvic floor muscle strength is significantly reduced three to eight days postpartum in women following vaginal birth but not in women after caesarean delivery. Six to ten weeks later palpation and vesical neck elevation on perineal ultrasound do not show any significant differences to antepartum values, while intravaginal pressure on perineometry remains significantly lower in primiparae, but not in multiparae.

Conclusions Pelvic floor muscle strength is impaired shortly after vaginal birth, but for most women returns within two months.

\section{INTRODUCTION}

Pelvic floor damage occurring during childbirth is known to play a role in causing urinary stress incontinence and pelvic organ prolapse $\mathrm{e}^{1,2}$. Neurophysiological studies have documented re-innervation phenomena on single fibre and concentric needle electromyography and prolongation of pudendal nerve terminal conduction velocity after vaginal birth ${ }^{3,4}$. However, demonstration of prior neurogenic damage does not necessarily indicate impaired muscle function since reinnervation and compensatory muscle hypertrophy may compensate for prior denervation ${ }^{5}$. Studies evaluating damage to the complex pelvic floor system after childbirth should include quantitative measurements of each aspect of pelvic floor function. One quantitative method is the assessment of levator ani muscle strength. Impairment of muscle strength is an indication of damage to the neuromuscular unit. The aim of this study was to evaluate pelvic floor muscle strength in nulliparous and parous women before and after vaginal delivery with palpation, perineometry and perineal ultrasound.

Correspondence: Dr U. M. Peschers, I. Frauenklinik der Universität, Maistraße 11, 80337 München, Germany.

\section{METHODS}

We recruited women who attended the antenatal clinic at the Department of Obstetrics and Gynaecology of the Cantonal Hospital Lucerne, Switzerland; 55 women gave informed consent to participate in the study, including 25 primiparae (mean age 28.2 years [SD 4.31 ]) and 20 parous women (mean age 31.9 years [SD 3.88]) with a median of one previous birth (range $0-4$ ). Ten women (mean age 30.2 years [SD 4.9]) who were scheduled for elective caesarean section acted as a control group. The control group included eight primiparae and two who previously had had an elective caesarean section without ever having gone into labour. Women were examined at the 36th to 42nd week of pregnancy (examination 1), three to eight days postpartum (examination 2) and six to ten weeks postpartum (examination 3).

Fifteen women (eight primiparae, mean age 28.3 years [SD 4.3], and seven multiparae, mean age 31.7 years [SD 4.1]) agreed to undergo a follow up examination (examination 4) after six to fifteen months (mean 11.4 months). The women were asked to attend with a comfortably full bladder; bladder volume was assessed by ultrasound. 


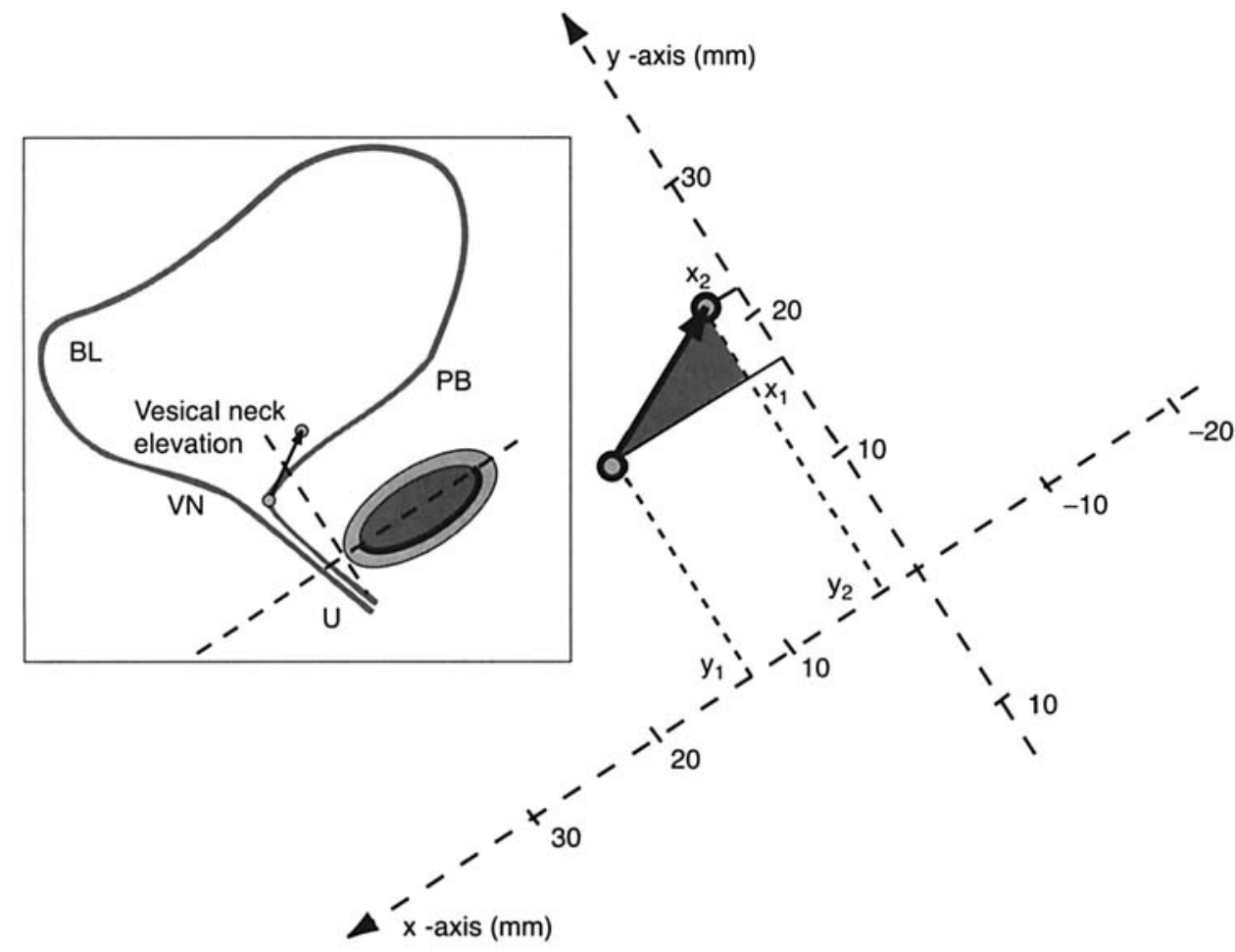

Fig. 1. Evaluation of bladder neck elevation during pelvic floor muscle contraction with perineal ultrasound. The $x, y$-coordinates of bladder neck position are established at rest and at maximum voluntary contraction of the pelvic floor muscles. The elevation of the vesical neck is measured by calculating a vector from the resting position $\left(x_{1}, y_{1}\right)$ to the position during contraction $\left(x_{2}, y_{2}\right)$ using the formula: vector length $=$ $\sqrt{ }\left\{\left(x_{1}-x_{2}\right)^{2}+\left(y_{1}-y_{2}\right)^{2}\right\}$.

\section{Assessment of pelvic floor muscle strength}

All examinations were performed with the woman in the lithotomy position. Detailed instructions on how to correctly squeeze the pelvic muscles were given to each woman. Then she was asked to contract the levator ani muscle three times as forcefully as possible; the strongest effort was taken for evaluation.

Vaginal palpation was graded with the Oxford Score, the standardisation and reproducibility of which have been reported previously ${ }^{6}$. The scale grades are:

$0=$ nil;

$1=$ flicker;

2 = weak;

$3=$ medium, slight lift of the examiner's finger, no resistance;

$4=$ strong, sufficient to elevate the examiner's finger against light resistance; and

$5=$ very strong, sufficient to elevate the examiner's finger against strong resistance.

Intravaginal pressure was measured with an air-filled perineometer (Cardio-Design, Brisbane, Australia); recordings were measured in centimetres of water $\left(\mathrm{cmH}_{2} \mathrm{O}\right)$. Perineometer readings were taken with the probe in a standardised position marked by the end of the probe being located $1.5 \mathrm{~cm}$ distal of the hymenal ring. To ensure that pressure was not generated purely by a straining effort, the position of the probe was controlled visually and women carefully observed to detect straining efforts.

Perineal ultrasound was performed using a Siemens SI 400 ultrasound machine (Siemens-Medical, Erlangen, Germany) with a $3.5 \mathrm{MHz}$ convex scanner with the women in lithotomy position. The scanner was positioned on the perineum and the pubic bone and the urethra were visualised. The ultrasound examination was recorded on video tape to allow subsequent detailed analysis.

Ultrasound measurements were made in a standardised method shown to have a high level of accuracy and reliability ${ }^{7}$. A reference co-ordinate system was determined with the $x$-axis coincident with the longitudinal axis of the pubic bone and $y$-axis intersecting at the inferior margin of the pubis (Fig. 1). Bladder neck position was evaluated at rest and and maximum contraction of the pelvic floor muscles and the distance between these two points was calculated.

Analysis of covariance was used to control for age and weight between groups. Wilcoxon signed rank test and Kruskal-Wallis one way analysis of variance were applied for statistical evaluation. Post hoc analysis was performed using the Bonferoni method of multiple comparison. 
Table 1. Pelvic floor muscle strength assessed by palpation, perineometry and perineal ultrasound at 36th to 42 nd week of pregnancy (Exam 1), 3-8 days postpartum (Exam 2$)$ and 6-10 weeks postpartum (Exam 3$)$ in primiparae $(n=25)$ and multiparae $(n=20)$ who had given birth vaginally, and 10 women acting as controls who had had elective caesarean section. Values are given as mean [SD]. Perineal US $=$ urethral elevation during pelvic floor muscle contraction on perineal ultrasound; NS = not significant.

\begin{tabular}{|c|c|c|c|c|c|c|}
\hline & Exam 1 & Exam 2 & Exam 3 & $P(1$ vs 2$)$ & $P(2 v s 3)$ & $P(1 \vee s 3)$ \\
\hline \multicolumn{7}{|l|}{ Primiparae } \\
\hline Palpation (score) & $3 \cdot 1[1 \cdot 6]$ & $1 \cdot 7[1 \cdot 3]$ & $2.9[1.4]$ & $<0.001$ & $<0.001$ & NS \\
\hline Perineometry $\left(\mathrm{cmH}_{2} \mathrm{O}\right)$ & $31 \cdot 3[16 \cdot 6]$ & $13 \cdot 0[9.6]$ & $20 \cdot 2[10 \cdot 6]$ & $<0.001$ & $<0.005$ & $<0.001$ \\
\hline Perineal US (mm) & $11 \cdot 8[6 \cdot 0]$ & $7 \cdot 5[5 \cdot 4]$ & $11.6[5.9]$ & $<0.004$ & $<0.002$ & NS \\
\hline \multicolumn{7}{|l|}{ Multiparae } \\
\hline Palpation (score) & $2.5[0.9]$ & $1.6[0.9]$ & $2.6[1.0]$ & $<0.004$ & $<0.002$ & NS \\
\hline Perineometry $\left(\mathrm{cmH}_{2} \mathrm{O}\right)$ & $26 \cdot 2[10 \cdot 5]$ & $15 \cdot 2[8 \cdot 9]$ & $24 \cdot 7[14 \cdot 7]$ & $<0.001$ & $<0.001$ & NS \\
\hline Perineal US (mm) & $10 \cdot 0[4 \cdot 5]$ & $7 \cdot 1[5 \cdot 3]$ & $12 \cdot 4[6 \cdot 9]$ & $<0.001$ & $<0.005$ & NS \\
\hline \multicolumn{7}{|l|}{ Control group } \\
\hline Palpation (score) & $3.4[0.9]$ & $3.2[0.7]$ & $3.4[0.9]$ & NS & NS & NS \\
\hline Perineometry $\left(\mathrm{cmH}_{2} \mathrm{O}\right)$ & $28 \cdot 7[15 \cdot 2]$ & $26 \cdot 2[11 \cdot 3]$ & $29 \cdot 7[15 \cdot 6]$ & NS & NS & NS \\
\hline Perineal US (mm) & $10 \cdot 0[2 \cdot 6]$ & $10 \cdot 6[4.4]$ & $9 \cdot 4[2.9]$ & NS & NS & NS \\
\hline
\end{tabular}

*Wilcoxon signed rank test.

\section{RESULTS}

Mean birthweight of infants was not different between groups: primiparae $3591 \mathrm{~g}$ [SD 519]; multiparae $3598 \mathrm{~g}$ [SD 422]; caesarean section $3583 \mathrm{~g}$ [SD 620]. The mean duration of the second stage of labour was longer in the primiparous group (60.5 min [SD 59.5]) compared with the multiparous group (20.1 $\mathrm{min}$ [SD 29.5]) as expected.

Bladder volume ranged from 50 to $400 \mathrm{~mL}$. The was no significant difference between mean bladder filling before and after birth (examination 1: $169 \mathrm{~mL}$ [SD 95]; examination 2: $164 \mathrm{~mL}$ [SD 91]; examination 3: $170 \mathrm{~mL}$ [SD 99]; examination 4: $163 \mathrm{~mL}$ [SD 97]). The maximum individual difference of bladder filling was $150 \mathrm{~mL}$. Pelvic floor muscle strength before (examination 1) and after childbirth (examinations 2 and 3 ) are summarised in Table 1.

Primiparas and multiparas who underwent vaginal delivery showed a significant reduction of pelvic floor muscle strength evaluated by palpation, perineometry and perineal ultrasound three to eight days after childbirth. However, six to ten weeks after delivery a significant increase of pelvic floor muscle strength could be demonstrated. At this time there were no significant mean differences with antepartum values except for perineometry in primiparae $\left(31.75 \mathrm{cmH}_{2} \mathrm{O}\right.$ [SD 16.6] versus $20.2 \mathrm{cmH}_{2} \mathrm{O}$ [SD 10.6]; $P<0.001$, Wilcoxon signed rank test). No significant changes in pelvic floor muscle strength were found in the control group.

\section{Follow up evaluation after one year}

Table 2 summarises the results obtained in the subgroup who returned for a follow up visit after a mean time of 11.4 months (range 9-15). There are no significant
Table 2. Pelvic floor muscle strength assessed by palpation, perineometry and perineal ultrasound at 6-10 weeks postpartum (Exam 3$)$ and $9-15$ months postpartum (Exam 4$)$ in primiparae $(n=$ 8 ) and multiparae $(n=7)$. Values are given as mean [SD]. No values were statistically significant. Key as for Table 1.

\begin{tabular}{|c|c|c|}
\hline & Exam 3 & Exam 4 \\
\hline \multicolumn{3}{|l|}{ Primiparae } \\
\hline Palpation (score) & $2.9[1.4]$ & $3 \cdot 2[1 \cdot 4]$ \\
\hline Perineometry $\left(\mathrm{cmH}_{2} \mathrm{O}\right)$ & $20 \cdot 3[12 \cdot 3]$ & $21.9[11 \cdot 3]$ \\
\hline Perineal US (mm) & $11 \cdot 3[6 \cdot 4]$ & $9 \cdot 8[4 \cdot 6]$ \\
\hline \multicolumn{3}{|l|}{ Multiparae } \\
\hline Palpation (score) & $2.9[1 \cdot 5]$ & $3 \cdot 1[1 \cdot 2]$ \\
\hline Perineometry $\left(\mathrm{cm} \mathrm{H}_{2} \mathrm{O}\right)$ & $24 \cdot 4[16 \cdot 1]$ & $24 \cdot 0[13 \cdot 4]$ \\
\hline Perineal US (mm) & $13 \cdot 0[7 \cdot 5]$ & $10 \cdot 9[5 \cdot 5]$ \\
\hline
\end{tabular}

changes comparing pelvic floor muscle strength 6-10 weeks postpartum to $9-15$ months postpartum.

\section{DISCUSSION}

This study shows that the ability to voluntarily contract the levator ani muscles is significantly reduced shortly after vaginal birth. Levator ani muscle strength is restored to antepartum values in the majority of women six to ten weeks postpartum.

Neurogenic damage to the pelvic floor at the time of childbirth was first documented by Snooks et al. ${ }^{1}$ in 1984. They found denervation of the external anal sphincter and suggested that repeated measurements in the individual women were the most reliable way to obtain data on the causality of changes ${ }^{1}$. Allen et al. ${ }^{3}$ reported that childbirth had resulted in neurogenic changes in 69 primiparous women with vaginal delivery who underwent neurophysiological assessment before and after vaginal birth, compared with a control group 
of women who had had elective caesarean section. However, it remains unclear to what extent the demonstrated neurogenic changes lead to impaired function, because the parameters studied demonstrated reinnervation patterns which could have been sufficient to compensate for prior denervation ${ }^{5}$. Some neurogenic changes can be demonstrated in the majority of parous women compared with nulliparas ${ }^{2}$, but urinary incontinence, faecal incontinence or prolapse develop only in some of these women.

The lack of direct association between neurophysiological test results and pelvic floor function underscores the necessity of obtaining quantitative data on the different aspects of pelvic floor function before and after childbirth. Sampselle ${ }^{8}$ measured pelvic floor muscle strength by palpation in a small group of primiparous women before and three months following vaginal delivery. An overall reduction of levator ani muscle strength after vaginal birth was reported. This study also indicated that the development of stress urinary incontinence was related to impaired contraction strength. The results of our study showed a significant reduction in palpated strength directly following vaginal birth, but measurements taken 6-10 weeks postpartum did not differ significantly from antepartum values. Although good reproducibility of the assessment of levator ani muscle strength was demonstrated by Laycock ${ }^{6}$ the scale of measurement contained only 6 grades which may present some difficulty in assessing slight differences in muscle strength.

Contrary to palpation, perineometry is an objective quantitative way to measure intravaginal pressure. Originally described by $\mathrm{Kegel}^{9}$ in 1948, perineometry is widely used for biofeedback and research ${ }^{10}$. The technique has been shown to have a strong reproducibility ${ }^{6}$. However, perineometry cannot differentiate between the pressure generated by a pelvic muscle contraction and intra-abdominal pressure. To avoid false measurements, it has been suggested ${ }^{11}$ that this technique should only be used in women in whom movement of the perineometer can be measured when the pelvic muscles are contracted. However, the problem of undifferentiated pressure measurements as a reflection of pelvic muscle force is not eliminated by this technique.

Allen et al. ${ }^{3}$ reported that pelvic muscle strength evaluated by perineometry was significantly reduced six weeks postpartum in primiparae. In contrast we found that only perineometry values were significantly reduced in primiparae, while palpation and bladder neck elevation on perineal ultrasound were unchanged. Multiparae did not show perineometric changes after vaginal childbirth. It is possible that in the primiparae described by Allen $e t a l^{3}$, as well as in the primiparous women in our study, the vagina might have been widened by vaginal birth, leading to a decreased pressure transmission to the probe, as the size of the probe remained unchanged. Whether increased widening of the vagina in women with the same level of pelvic floor muscle strength leads to lower perineometer readings (if the size of the probe remains unchanged) requires further investigation.

The measurement of bladder neck elevation with perineal ultrasound indicates the effect of a levator ani muscle contraction on bladder neck position. Schaer $e t$ $a l^{7}$ standardised the evaluation system used and demonstrated its reproducibility. In this study, bladder neck position was assessed at rest and at contraction of the levator ani muscles. The elevation of the bladder neck was calculated as the vector between these positions. In previous studies we have shown that bladder neck elevation at contraction is lower in nulliparae than in primiparae ${ }^{12}$. This indicates that bladder neck elevation is somehow dependent on the resting position, but the exact relation between these two variables remains unclear. This technique therefore only allows indirect conclusions on pelvic floor muscle strength. However, as increases in intra-abdominal pressure lead to a descent rather than to an elevation of the bladder neck, it is possible to quantify the effect of a levator ani muscle contraction.

Bladder volume was not standardised in this study. Women were asked to come in with the bladder comfortably full. Differences in bladder volume of up to $150 \mathrm{~mL}$ were found. Schaer et al. ${ }^{13}$ have demonstrated that bladder volume does not have a significant impact on bladder neck mobility. Unpublished data of our own have not demonstrated significant changes in perineometer readings and palpated pelvic floor muscle strength grading in a group of 42 nulliparous women with a full and an empty bladder.

The impact of vaginal delivery on pelvic muscle strength appears to vary according to the time of postpartum assessment. We performed early postpartum examinations in order to describe changes occurring immediately after childbirth. However, even though the influence of pain on the compliance of the women was not systematically evaluated in this study, it became clear that many women were either unable or unwilling to attempt maximum contractions because of discomfort. The early postpartum evaluation therefore does not seem to provide reliable information of the women's maximum pelvic floor muscle strength. The timing of the second postpartum examination in this study was selected mainly on the basis of convenience for the women, because it was combined with their routine postpartum appointment. The finding that there were no notable changes within patient subgroups who returned for a follow up after one year indicates that the evaluation of the postpartum status of the pelvic floor at the routine postpartum appointment is appropriate. 


\section{References}

I Snooks SJ, Setchell M, Swash M, Henry MM. Injury to the innervation of the pelvic floor sphincter musculature in childbirth. Lancet $1984 ; 2: 546-550$

2 Smith ARB, Hosker GL, Warrell DW. The role of partial denervation of the pelvic floor in the aetiology of genitourinary prolapse and stress incontinence of urine. Br J Obstet Gynaecol 1989; 96: 24-28.

3 Allen RE, Hosker GL, Smith ARB, Warrell DW. Pelvic floor damage in childbirth: a neurophysiological study. Br J Obstet Gynaecol 1990, 97: $770-779$.

4 Sultan AH, Kamm MA, Hudson CN. Pudendal nerve damage during labour: prospective study before and after childbirth. $\mathrm{Br} J$ Obstet Gynaecol 1994; 101: 22-28.

5 Vodusek D. Arguments against the neurogenic hypothesis of stress incontinence. International Continence Survey 1994; 4: 11-15.

6 Laycock J. Pelvic Floor Dysfunction. PhD thesis; Bradford University; 1995.

7 Schaer GN, Koechli OR, Schuessler B, Haller U. Perineal ultrasound for evaluating the bladder neck in urinary stress incontinence. Obstet Gynecol 1995; 85: 220-224.

8 Sampselle CM. Changes in pelvic floor muscle strength associated with childbirth. J Obstet Gynecol Neonatal Nurs 1990; 19: 371-377.
9 Kegel AH. Physiologic therapy for urinary stress incontinence. JAMA 1951; 146: 915-917.

10 Laycock J. Biofeedback control. In: Schuessler B, Laycock J, Norton P, Stanton S, editors. Pelvic Floor Re-education. London: Springer Verlag, 1994: 153-156.

11 Bo K, Kvarstein B, Hagen RR, Larsen S. Pelvic floor muscle exercises for the treatment of female stress urinary incontinence: II. Neurourol Urodynam 1990; 9: 479-487.

12 Peschers U, Schaer GN, Anthuber C, DeLancey JOL, Schuessler B. Changes in vesical neck mobility following vaginal delivery. Obstet Gynecol 1996; 88: 1001-1006.

13 Schaer GN, Koechli OR, Schuessler B, Haller U. Perineal ultrasound: determination of reliable examination procedures. Ultrasound Obstet Gynecol 1996; 7: 347-352.

Received 25 September 1996

Returned for revision 21 November 1996 and 1 April 1997 Accepted 27 May 1997 